\title{
The impact of a disease management programme for type 2 diabetes on health-related quality of life: multilevel analysis of a cluster-randomised controlled trial
}

\author{
Sigrid Panisch ${ }^{1 \dagger}$, Tim Johansson ${ }^{2 \dagger}$, Maria Flamm² ${ }^{2}$, Henrike Winkler ${ }^{3}$, Raimund Weitgasser ${ }^{4,5}$
} and Andreas C. Sönnichsen ${ }^{6,7^{*}}$

\begin{abstract}
Background: Type 2 diabetes is a chronic disease associated with poorer health outcomes and decreased health related quality of life (HRQoL). The aim of this analysis was to explore the impact of a disease management programme (DMP) in type 2 diabetes on HRQoL. A multilevel model was used to explain the variation in EQ-VAS.

Methods: A cluster-randomized controlled trial — analysis of the secondary endpoint HRQoL. Our study population were general practitioners and patients in the province of Salzburg. The DMP "Therapie-Aktiv" was implemented in the intervention group, and controls received usual care. Outcome measure was a change in EQ-VAS after 12 months. For comparison of rates, we used Fisher's Exact test; for continuous variables the independent T test or Welch test were used. In the multilevel modeling, we examined various models, continuously adding variables to explain the variation in the dependent variable, starting with an empty model, including only the random intercept. We analysed random effects parameters in order to disentangle variation of the final EQ-VAS.

Results: The EQ-VAS significantly increased within the intervention group (mean difference 2.19, $p=0.005$ ). There was no significant difference in EQ-VAS between groups (mean difference 1.00, $p=0.339$ ). In the intervention group the improvement was more distinct in women $(2.46, p=0.036)$ compared to men $(1.92, p=0.063)$. In multilevel modeling, sex, age, family and work circumstances, any macrovascular diabetic complication, duration of diabetes, baseline body mass index and baseline EQ-VAS significantly influence final EQ-VAS, while DMP does not. The final model explains 28.9\% (EQ-VAS) of the total variance. Most of the unexplained variance was found on patient-level (95\%) and less on GP-level (5\%).
\end{abstract}

Conclusion: DMP "Therapie-Aktiv" has no significant impact on final EQ-VAS. The impact of DMPs in type 2 diabetes on HRQoL is still unclear and future programmes should focus on patient specific needs and predictors in order to improve HRQoL.

Trial registration Current Controlled trials Ltd., ISRCTN27414162

Keywords: Cluster randomised controlled trial, Type 2 diabetes mellitus, Disease management programme, Health related quality of life (EQ-5D-3L questionnaire), Multilevel modeling, Sex-specific analysis

\footnotetext{
*Correspondence: andreas.soennichsen@uni-wh.de

${ }^{+}$Sigrid Panisch and Tim Johansson contributed equally to this work

${ }^{6}$ Institute of General Practice and Family Medicine, Witten/Herdecke

University, Alfred-Herrhausen-Straße 50, 58448 Witten, Germany

Full list of author information is available at the end of the article
}

(c) The Author(s) 2018. This article is distributed under the terms of the Creative Commons Attribution 4.0 International License (http//creativecommons.org/licenses/by/4.0/), which permits unrestricted use, distribution, and reproduction in any medium, provided you give appropriate credit to the original author(s) and the source, provide a link to the Creative Commons license, and indicate if changes were made. The Creative Commons Public Domain Dedication waiver (http://creativecommons.org/ publicdomain/zero/1.0/) applies to the data made available in this article, unless otherwise stated. 


\section{Background}

The prevalence of type 2 diabetes is increasing and the social and economic burden of this disease grows rapidly [1, 2]. Guideline adherent, structured treatment and management of the disease as proposed by DMPs are the best strategies to prevent acute and chronic complications, while preserving a good quality of life $[3,4]$. Several studies show that the quality of life in diabetes is decreased compared to individuals without diabetes [5] and HRQoL decreases with disease progression and complications [6,7]. Predictive factors for HRQoL are age, sex, low socioeconomic status (family and work circumstances, level of education, residential area), ethnicity, obesity, comorbidities, and any macrovascular diabetic complication [5, 7-12]. The prevalence of type 2 diabetes is higher among women than among men. A recent meta-analysis demonstrated that women are at higher risk of developing both coronary heart disease and stroke compared to men [13, 14]. Increasing knowledge concerning $\mathrm{HRQ}$ oL in diabetic patients, as well as the predictors, is crucial in improving diabetes management. Overall, evidence from RCTs and systematic reviews on DMPs reveal only modest effects on patient care [3, 4], especially regarding improvement in clinically relevant endpoints such as HRQoL. We previously demonstrated that the Austrian DMP “Therapie-Aktiv" implemented by statutory health insurance improves process quality and enhances weight reduction, but did not significantly improve metabolic control (HbA1c) [15]. Participants in the German DMP for type 2 diabetes rated their HRQoL in the dimensions mobility, self-care and performing usual activities higher compared to patients in routine care [16]. Although studies are reporting the effect of DMPs on quality of life in diabetes patients [17-20], none of these interventions was designed as a structured, long-term programme implemented by statutory health insurance and conducted and evaluated as a randomized controlled trial. Enhancement of the understanding of HRQoL in diabetes and related risk factors is of great importance. We therefore studied HRQoL in patients with type 2 diabetes participating in the Austrian DMP "Therapie-Aktiv".

\section{Methods}

\section{Study aim}

The aim of this study was to explore the impact of the Austrian DMP “Therapie-Aktiv" on HRQoL using the individual EQ-5D VAS and EQ-5D index in patients with type 2 diabetes. A multilevel model was used to identify variables that influence EQ-VAS and to assess its variation between patients, GPs and districts including a sex-specific analysis.

\section{Study design}

This work was based on a pragmatic cluster-randomized controlled trial of the Austrian DMP "Therapie-Aktiv" in the province of Salzburg, Austria. The trial was approved by the ethics committee of Salzburg and registered with current controlled trials Ltd. (ISRCTN27414162) on July 12, 2007. The methodology was described in our study protocol [21] and the main findings (significant improvement of process quality but no significant change in metabolic control after 1 year) have been published previously [15].

\section{Setting of the study and characteristics of participants}

Participation in the study was offered to all 275 family physicians and specialists for internal medicine having a contract with the Salzburg statutory health insurance. All patients ( $>18$ years) with type 2 diabetes that fulfilled the WHO/ADA-criteria for diabetes diagnosis were eligible to participate in the study. The recruitment period was July 1 st to October 31 st of 2007 . Exclusion criteria were dementia/psychiatric illness with inability to participate or to give informed consent, or known major consuming illness (i.e. advanced cancer).

\section{Intervention}

The Austrian DMP for type 2 diabetes "Therapie-Aktiv" [22] was developed in 2004 by the Austrian statutory health insurance. The programme aims to prevent diabetes complications and to improve quality of life through: (1) prevention and health promotion; (2) structured diagnosis and medical treatment; (3) considering patients' overall cardiovascular risk; and (4) stronger patient involvement. Patients must sign a participant consent form to be enrolled in the programme. The attending GPs administer the patient informed consent. Patients participate in an education course on type 2 diabetes where they learn how to manage their disease. They receive a "patient's booklet" which deals with topics such as healthy lifestyle (e.g. nutrition, exercise), blood glucose management, diabetes medication, and preventable long term complications. "Therapie-Aktiv" includes five main components:

(1) Physician training consisting of an obligatory 10-h face to face course. This training was designed by the Austrian Diabetes Association, the Austrian Medical College and the Austrian Society for General Practice. It comprised an update in diabetes care, current guidelines of the Austrian Diabetes Association and practice management training;

(2) Patient education consisting of $9 \mathrm{~h}$ face to face courses in small groups. The patient education was organized by the Working Group for Preventive 
Medicine Salzburg (Arbeitskreis Vorsorgemedizin Salzburg) using the "Düsseldorfer Modell" curriculum [23];

(3) Quarterly patient-physician encounters of 15-20 min duration to discuss the results of laboratory tests (HbA1c, eGFR, microalbumine) and physical examination (BMI, blood pressure, foot examination, neurological examination) and determine treatment goals for the next quarter year, including standardised documentation of physical examination, laboratory findings and diabetes complications in a DMP case report form once a year [22];

(4) Structured interdisciplinary care according to the guidelines of the Austrian Diabetes Association [24]; and

(5) Agreement on therapeutic goals in a shared patient-physician decision-making process. These agreements are signed by the patient and physician every third month.

In the control group, physicians performed usual care.

\section{Data collection}

Baseline and follow-up data were collected by the responsible GP. Standardised documentation of physical examination and laboratory findings in a DMP-form was performed once a year. The DMP form was administered in both study groups. Patients' education and occupational status, smoking status, living situation, nationality, macrovascular diabetic complications (i.e. myocardial infarction, PTCA/stenting, coronary bypass, stroke, carotid surgery, amputation/gangrene, peripheral artery bypass or PTA), and self-rated HRQoL (EQ-5D-3L) were assessed by a standardised questionnaire. Follow-up data were collected after 12 months intervention.

\section{Outcomes and statistical analysis}

We used the EQ-5D-3L to evaluate the self-rated HRQoL $[25,26]$. The EQ-5D-3L consists of two parts: the EQ-5D descriptive system and the EQ visual analogue scale (EQVAS). The EQ-5D-3L descriptive system comprises five dimensions: mobility, self-care, usual activities, pain/ discomfort and anxiety/depression. Each dimension has three levels: no problems, some problems, extreme problems. The EQ-index score is calculated based on the results of the five dimensions by using a scoring algorithm. The EQ-VAS records the respondent's self-rated health on a vertical thermometer-like scale $(0-100)$ where the endpoints are labelled worst imaginable health state' and 'best imaginable health state'. Following the user's guide of the EuroQol Group [26], ambiguous scores were treated as missing. Results were reported according to recent guidelines [27]. For comparison of rates, we used Fisher's Exact Test; for continuous variables we used the independent T-Test or Welch-Test. To assess changes over the study period of 1 year and in order to find factors that influence HRQoL outcomes, we analysed changes of EQ-VAS and EQ-index within and between groups, using repeated measures general linear model. Patients with at least 300 days of study duration and with two valid EQ-VAS scores (baseline and followup) were included in the analysis. In order to evaluate the final HRQoL we identified variables that explain some of the variation in final EQ-VAS and EQ-index as well as to assess their variation at different levels within a multilevel framework. We further incorporated sex-specific variables and examined the effect of living situation (living alone or not), education and occupational status (fulltime work or not), nationality (Austrian or not), any manifestation of coronary heart disease (myocardial infarction and/or PTCA/stenting and/or coronary bypass), any macrovascular diabetic complication (myocardial infarction and/or PTCA/stenting and/or coronary bypass and/ or stroke and/or carotid surgery and/or amputation/gangrene and/or peripheral artery bypass or PTA) as they are known to potentially influence HRQoL within a diabetic population. We analysed educational level as low (no school leaving examination) or high (school leaving examination or higher). A full guideline adherence treatment covered four aspects: patient education, diagnostic measures (i.e. regular HbA1c-checks), ophthalmological examination, and foot examinations.

The multilevel modeling takes three levels into account: patient-level (sex, age, nationality, education, occupational, family and smoking status, study length), GP-level (intervention or control group), and district-level ("rural", "urban" or "mixed"). We started modeling patients' HRQoL by separating all three sources of variation. Hence, our multilevel linear model can be written as follows: $y_{i j k}=\beta_{0 i j k}+\boldsymbol{\beta} \boldsymbol{X}_{i j k}+\boldsymbol{\gamma} \boldsymbol{Y}_{j k}+\delta \boldsymbol{Z}_{k}$, where $y_{i j k}$ represents the dependent variable, i.e. final EQ-VAS or final EQ-index, that is a function of explanatory variables on patient-level $(\mathbf{X})$, GP-level $(\mathbf{Y})$ and district-level $(\mathbf{Z})$ with corresponding coefficients $\boldsymbol{\beta}, \boldsymbol{\gamma}$ and $\boldsymbol{\delta}$, respectively. The overall error term $\beta_{0 i j k}$ can be decomposed into $\beta_{0}+v_{0 k}+u_{0 j k}+e_{0 i j k}$, where $v_{0 k}$ is the random error term for the kth district, $u_{0 j k}$ denotes the GP effect (of the jth GP within the kth district) and $e_{0 i j k}$ denotes the patient residual (error term of ith patient treated by the jth GP within the kth district). We examined various models, continuously adding variables to explain the variation in the dependent variable, starting with an empty model, including only the random intercept. We analysed random effects parameters in order to disentangle variation 


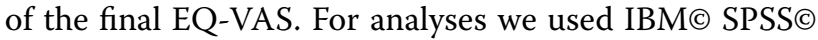
Statistics Version 19 and MLWiN 2.13 [28].

\section{Results}

\section{Characteristics of the study population}

Ninety-two (33.5\%) physicians participated and recruited 1489 patients in the province of Salzburg, Austria. Three hundred fifty patients were excluded from analysis (328 patients did not report two valid EQ-VAS scores and further 22 patients had a study period of less than 300 days). No significant differences were shown in baseline characteristics between included $(n=1139)$ and excluded $(\mathrm{n}=350)$ patients. The mean length of the study period was 398 days (SD 42). EQ-VAS was available in 512 (78.9\%) participants in the intervention group and in 627 (74.6\%) controls (Fig. 1). There were no relevant demographic differences between intervention and control group at baseline (Additional files 1 and 2). There were significant differences in baseline data disaggregated by sex (Table 1). In various subgroups there were significant differences in EQ-VAS (Table 2), EQ-index, and EQ five dimensions (Additional files 3, 4, 5).

\section{Outcomes-univariate analysis}

In univariate analysis, the EQ-VAS improved within both groups. It increased by 2.19 points $(\mathrm{p}=0.005)$ in the intervention group and by 1.18 points $(\mathrm{p}=0.094)$ in controls. The mean difference (1.00) between intervention and control group was not significant $(\mathrm{p}=0.339)$. In the intervention group the improvement was stronger in women $(2.46, \mathrm{p}=0.036)$ compared to men (1.92, $\mathrm{p}=0.063)$. Significant differences in EQ-VAS scores were found in various subgroups within the intervention group (Table 3). The EQ-index score hardly changed during the study period, so no multilevel analysis was performed for the EQ-index. The mean difference within the intervention group was $0.00(\mathrm{p}=0.612)$ (Additional file 6).

\section{Outcomes-multilevel analysis}

Most of the variance was found on patient-level (approximately 95\%). The GP-level (intervention or control group) and district-level ("rural", "urban" or "mixed") had only a modest $(5 \%)$ or no impact $(0 \%)$. Due to the negligible impact of district-level and the restricted number of districts $(n=6)$, we decided to continue with two levels, GPlevel and patient-level. The results of the empty model ("2-level null model") and the baseline-adjusted model for two levels ("baseline-adjusted") are presented in Table 4. Adding the baseline score accounted for $27.0 \%$ of the within GP-level variability in the EQ-VAS final score, and $30.9 \%$ of the variation in means across GPs could be attributed to differences in the baseline score of patients nested in these GP-surgeries. We continued adding variables in the fixed part of the model to examine variation in final EQ-VAS. First, we included patient characteristics (sex, age, nationality, education, occupational, family and smoking status, and study duration) as well as group association. The parameter estimate for study duration was negligible (0.01, SE 0.01), hence we present the results of this model ("socio-economic") without adjusting for study length. Age, living alone, female sex and baseline EQ-VAS have a significant and negative impact on final EQ-VAS. The enrolment in the DMP had no significant impact on final EQ-VAS $(p=0.646)$. Adding diabetes associated parameters, led to a slight decrease of unexplained variance. In patients with any macrovascular diabetic complication and longer diabetes duration the final EQ-VAS was significantly reduced $(p=0.021$ and $p=0.002$, respectively). Any manifestation of coronary heart disease had no significant impact on the final EQ-VAS. Subsequently, we adjusted for significant baseline differences (cholesterol, BMI) and guideline adherence. The addition of further variables consecutively reduced the amount of unexplained variance. However, the allocation of variation did not change much. In all models, the intraclass correlation coefficient on GPlevel amounted to approximately $5 \%$. Since baseline EQVAS was significantly related to the dependent variable, we checked whether the slope varied randomly across GPs. However, adding the random slope hardly changed parameter estimates and slope variance was not significant. Composition effects did not exist for EQ-VAS. In the final model, the final EQ-VAS score in the intervention group was on average 1.17 points higher compared to controls (while retaining all variables unchanged), however not significant $(\mathrm{p}=0.390)$.

The final model explains $28.9 \%$ (EQ-VAS) of the total variance. Our multilevel analysis demonstrates that approximately $95 \%$ of the residual variation in final EQVAS is attributable to patient-level, with baseline EQVAS showing to be positively associated with the final score. Most of the unexplained variance can be found on patient-level (approximately 95\%) and less on GP-level (approximately $5 \%$ ).

\section{Discussion}

\section{Main findings}

This is the first study analysing the impact of a DMP implemented by statutory public health insurance on HRQoL in patients with type 2 diabetes in a cluster randomised controlled trial. A further aim was to evaluate HRQoL within a multilevel framework as a result of the cluster-design. A special focus was put on a sex-specific analysis.

The EQ-VAS significantly increased within the intervention group and the improvement was stronger in 


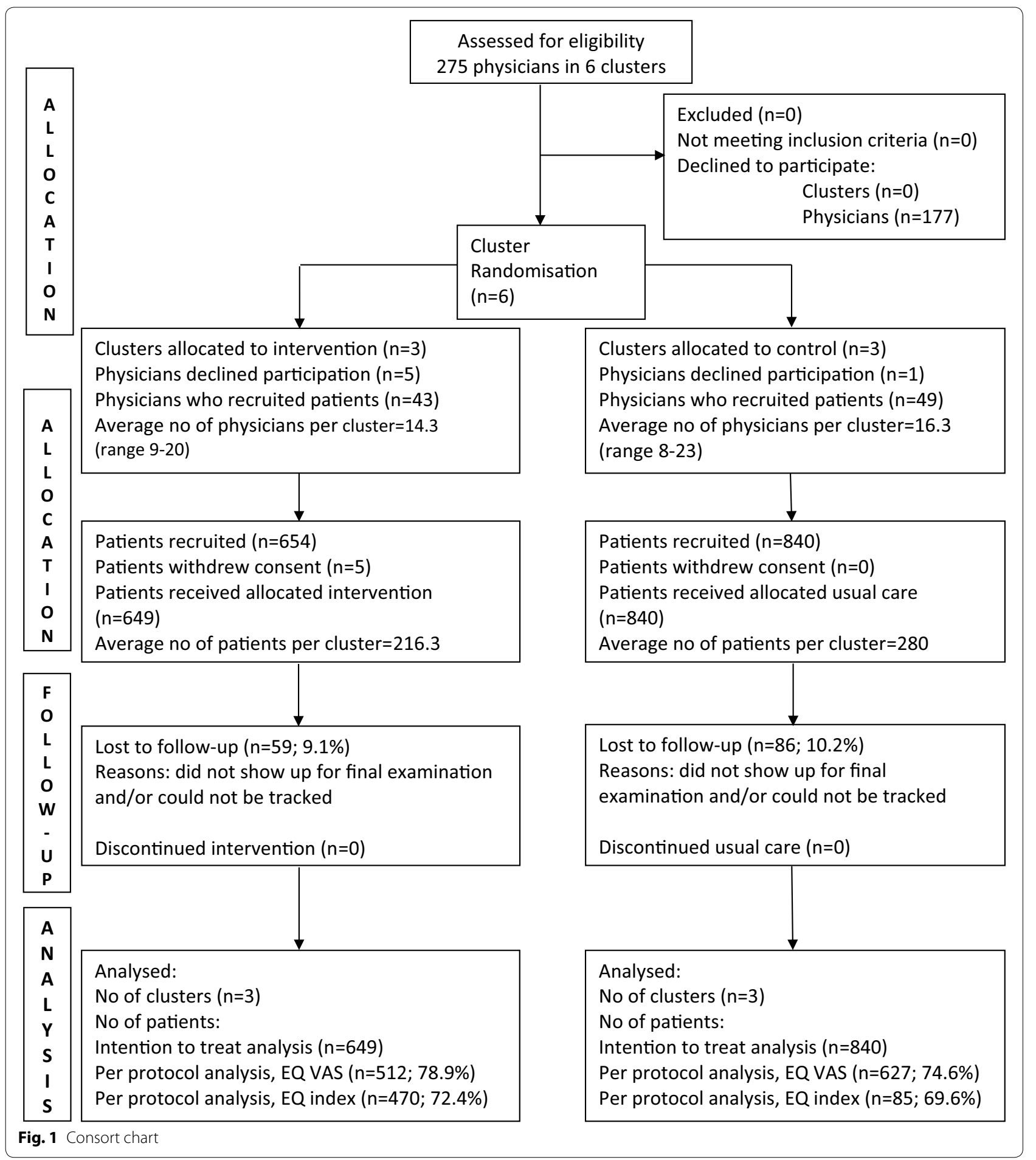

women than in men. This effect was not present when comparing mean differences between the intervention and control group. This may at least partly be due to a Hawthorne-effect in the control group mitigating the success of the intervention, but even when taking this into account the effect of the DMP on HRQoL is small and hardly of clinical relevance (see discussion of minimal important difference of EQ-VAS below). As the DMP also did not have a significant effect on metabolic control, and the observation period of one year was too short to detect 
Table 1 Baseline characteristics disaggregated by sex (per protocol analysis population)

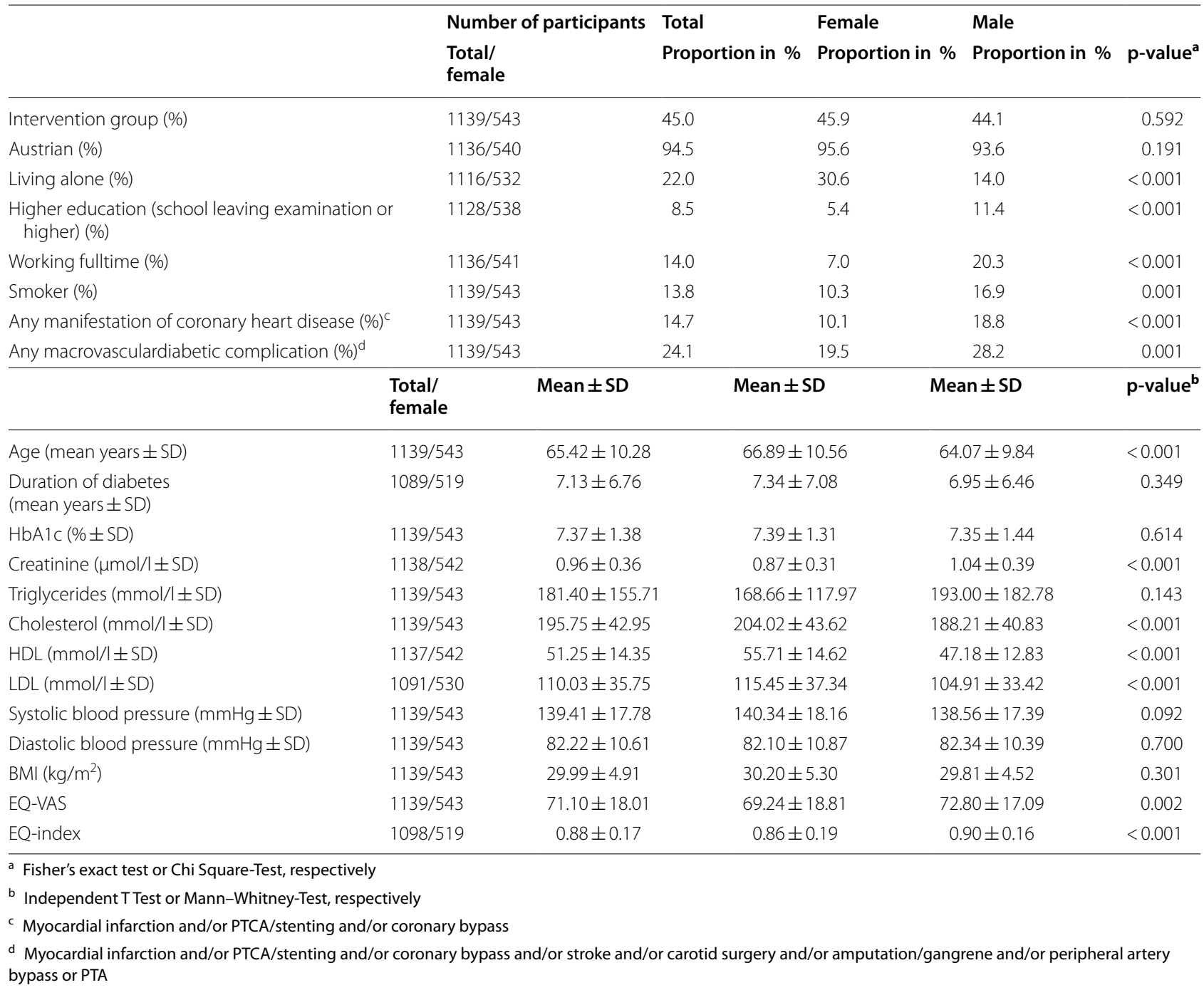

any effect regarding clinical endpoints, a large impact on HRQoL of this DMP-programme directed mainly at the improvement of process quality may not be expected. In our multilevel model we demonstrated that sex, age, family and work circumstances, any macrovascular diabetic complication, duration of diabetes, baseline body mass index and baseline EQ-VAS significantly influence EQVAS at follow-up, while DMP "Therapie-Aktiv" does not. Our results confirm that effects are easily overestimated in the absence of a control group, as also shown in a previous study [29]. While changes showed to be significant in many subpopulations of the intervention group, this effect disappeared after putting it in relation to the control group. Significant differences were found in the baseline data disaggregated by sex (Table 1) and in various subgroups (Table 2). For the EQ-VAS, men reported to have better HRQoL in comparison to women. However, some of the differences might be affected by social desirability bias: men are probably less likely to admit deficits in quality of life than women. In pre-post analysis, DMP patients with higher education exhibited the greatest improvement, whereas higher educated controls showed the worst development among all subgroups. Considering these results, the design and development of effective DMPs remains an important-yet open-issue [17, 30], and it may be concluded that "Therapie aktiv" needs to be intensified and targeted at outcome quality to be effective.

When analysing our results it also has to be kept in mind that although a multilevel model was used to assess final EQ-VAS variation between patients, GPs and districts, almost all variation (approximately 95\%) in final EQ-VAS was attributable to variables at the patient-level (e.g. age, sex, family and occupational status, duration of diabetes, baseline BMI and baseline EQ-VAS). 
Table 2 EQ-VAS at baseline, values for selected subgroups (intention-to-treat analysis population)

\begin{tabular}{|c|c|c|c|c|}
\hline Subgroup 1/subgroup 2 & $\begin{array}{l}\text { No of participants, subgroup 1/sub- } \\
\text { group } 2\end{array}$ & $\begin{array}{l}\text { EQ-VAS, } \\
\text { mean }[\mathrm{CI}] \text {, subgroup } 1\end{array}$ & $\begin{array}{l}\text { EQ-VAS, } \\
\text { mean }[\mathrm{CI}] \text {, subgroup } 2\end{array}$ & $p_{\text {value }}{ }^{a}$ \\
\hline Overall study population & 1464 & $70.1[69.1 ; 71.0]$ & & - \\
\hline Intervention/control & $639 / 825$ & $69.4[68.0 ; 70.8]$ & $70.6[69.3 ; 71.8]$ & 0.231 \\
\hline Female/male & $700 / 764$ & $68.4[67.0 ; 69.8]$ & $71.6[70.3 ; 72.8]$ & 0.001 \\
\hline $\begin{array}{l}\text { No manifestation of coronary heart } \\
\text { disease } \mathrm{e}^{\mathrm{b}} \text {. } \\
\text { any manifestation of coronary heart disease }\end{array}$ & $1253 / 211$ & $70.6[69.6 ; 71.6]$ & $66.6[64.1 ; 69.2]$ & 0.003 \\
\hline $\begin{array}{l}\text { No macrovascular diabetic complicationc/ } \\
\text { any macrovascular diabetic complication }\end{array}$ & $1108 / 356$ & $71.4[70.3 ; 72.4]$ & $66.0[64.1 ; 68.0]$ & $<0.001$ \\
\hline $\begin{array}{l}\text { Living with a partner/ } \\
\text { living alone }\end{array}$ & $1111 / 318$ & $71.0[69.9 ; 72.0]$ & $66.8[64.8 ; 68.8]$ & $<0.001$ \\
\hline Non Austrian/Austrian & $80 / 1377$ & $65.1[60.6 ; 69.7]$ & $70.3[69.4 ; 71.3]$ & 0.029 \\
\hline $\begin{array}{l}\text { No higher education/ } \\
\text { higher education }\end{array}$ & $1327 / 117$ & $69.9[68.9 ; 70.9]$ & $72.1[68.9 ; 75.4]$ & 0.202 \\
\hline $\begin{array}{l}\text { Working fulltime/ } \\
\text { not working fulltime }\end{array}$ & $1255 / 202$ & $69.7[68.7 ; 70.7]$ & $72.4[69.9 ; 74.9]$ & 0.050 \\
\hline
\end{tabular}

a Independent T Test or Welch-Test, respectively

b Myocardial infarction and/or PTCA/stenting and/or coronary bypass

c Myocardial infarction and/or PTCA/stenting and/or coronary bypass and/or stroke and/or carotid surgery and/or amputation/gangrene and/or peripheral artery bypass or PTA

Table 3 EQ-VAS in the intervention group at follow-up, various subgroups disaggregated by sex

\begin{tabular}{|c|c|c|c|c|c|c|}
\hline \multirow{3}{*}{$\begin{array}{l}\text { Total } \\
\text { Subgroups }\end{array}$} & \multirow{3}{*}{$\begin{array}{l}\text { Number of participants } \\
512 \\
\text { Female/male }\end{array}$} & \multirow{2}{*}{\multicolumn{2}{|c|}{$\begin{array}{l}\text { EQ-VAS, mean differ- } \\
\text { ence } \pm \text { SD } \\
2.19 \pm 17.55\end{array}$}} & \multirow{2}{*}{\multicolumn{3}{|c|}{$\begin{array}{l}\mathrm{p} \text { value } \\
0.005\end{array}$}} \\
\hline & & & & & & \\
\hline & & Female & Male & p value ${ }^{a}$ & p value ${ }^{b}$ & p value \\
\hline Female/male & $249 / 263$ & $2.46 \pm 18.46$ & $1.92 \pm 16.68$ & 0.005 & 0.729 & $<0.001$ \\
\hline Non Austrian & $10 / 15$ & $1.50 \pm 24.50$ & $-0.33 \pm 27.12$ & 0.914 & 0.865 & 0.490 \\
\hline Austrian & $239 / 248$ & $2.50 \pm 18.23$ & $2.06 \pm 15.91$ & 0.003 & 0.776 & 0.029 \\
\hline Living with a partner & $159 / 223$ & $2.48 \pm 17.95$ & $2.25 \pm 16.59$ & 0.008 & 0.897 & 0.002 \\
\hline Living alone & $85 / 35$ & $2.81 \pm 19.77$ & $-0.71 \pm 17.94$ & 0.589 & 0.364 & 0.425 \\
\hline High education (school leaving examination or higher) & $17 / 32$ & $3.53 \pm 18.18$ & $3.47 \pm 17.57$ & 0.196 & 0.991 & 0.073 \\
\hline No higher education & $229 / 229$ & $2.28 \pm 18.61$ & $1.77 \pm 16.64$ & 0.014 & 0.755 & 0.002 \\
\hline Not working fulltime & $227 / 211$ & $2.30 \pm 18.51$ & $2.73 \pm 16.15$ & 0.003 & 0.796 & 0.001 \\
\hline Working fulltime & $21 / 52$ & $3.81 \pm 18.63$ & $-1.37 \pm 18.47$ & 0.611 & 0.283 & 0.046 \\
\hline Non-smoker & $222 / 221$ & $2.18 \pm 18.09$ & $2.53 \pm 16.28$ & 0.004 & 0.831 & $<0.001$ \\
\hline Current smoker & $27 / 42$ & $4.74 \pm 21.52$ & $-1.29 \pm 18.54$ & 0.481 & 0.220 & 0.122 \\
\hline No manifestation of coronary heart disease ${ }^{d}$ & $219 / 216$ & $2.77 \pm 18.23$ & $1.69 \pm 17.51$ & 0.010 & 0.528 & $<0.001$ \\
\hline Any manifestation of coronary heart disease ${ }^{d}$ & $30 / 47$ & $0.23 \pm 20.22$ & $3.02 \pm 12.26$ & 0.381 & 0.453 & 0.410 \\
\hline No macrovascular diabetic complication ${ }^{e}$ & 189/192 & $3.06 \pm 17.39$ & $2.11 \pm 17.16$ & 0.004 & 0.592 & 0.001 \\
\hline Any macrovascular diabetic complication ${ }^{e}$ & $60 / 71$ & $0.58 \pm 21.53$ & $1.42 \pm 15.41$ & 0.537 & 0.796 & 0.022 \\
\hline Non guideline adherence treatment ${ }^{f}$ & $130 / 134$ & $2.22 \pm 17.34$ & $1.21 \pm 17.44$ & 0.110 & 0.636 & 0.004 \\
\hline Full guideline adherence treatment ${ }^{f}$ & $119 / 129$ & $2.72 \pm 19.68$ & $2.67 \pm 15.89$ & 0.018 & 0.980 & 0.012 \\
\hline
\end{tabular}

a Within-subject factor (time)

b Within-subject factor (time*sex)

c Between subject factors (sex)

${ }^{d}$ Myocardial infarction and/or PTCA/stenting and/or coronary bypass

${ }^{\text {e }}$ Myocardial infarction and/or PTCA/stenting and/or coronary bypass and/or stroke and/or carotid surgery and/or amputation/gangrene and/or peripheral artery bypass or PTA

${ }^{f}$ A full guideline adherence treatment covered four aspects: patient education; diagnostic measures (i.e. regular HbA1c-checks); ophthalmological and foot examinations 


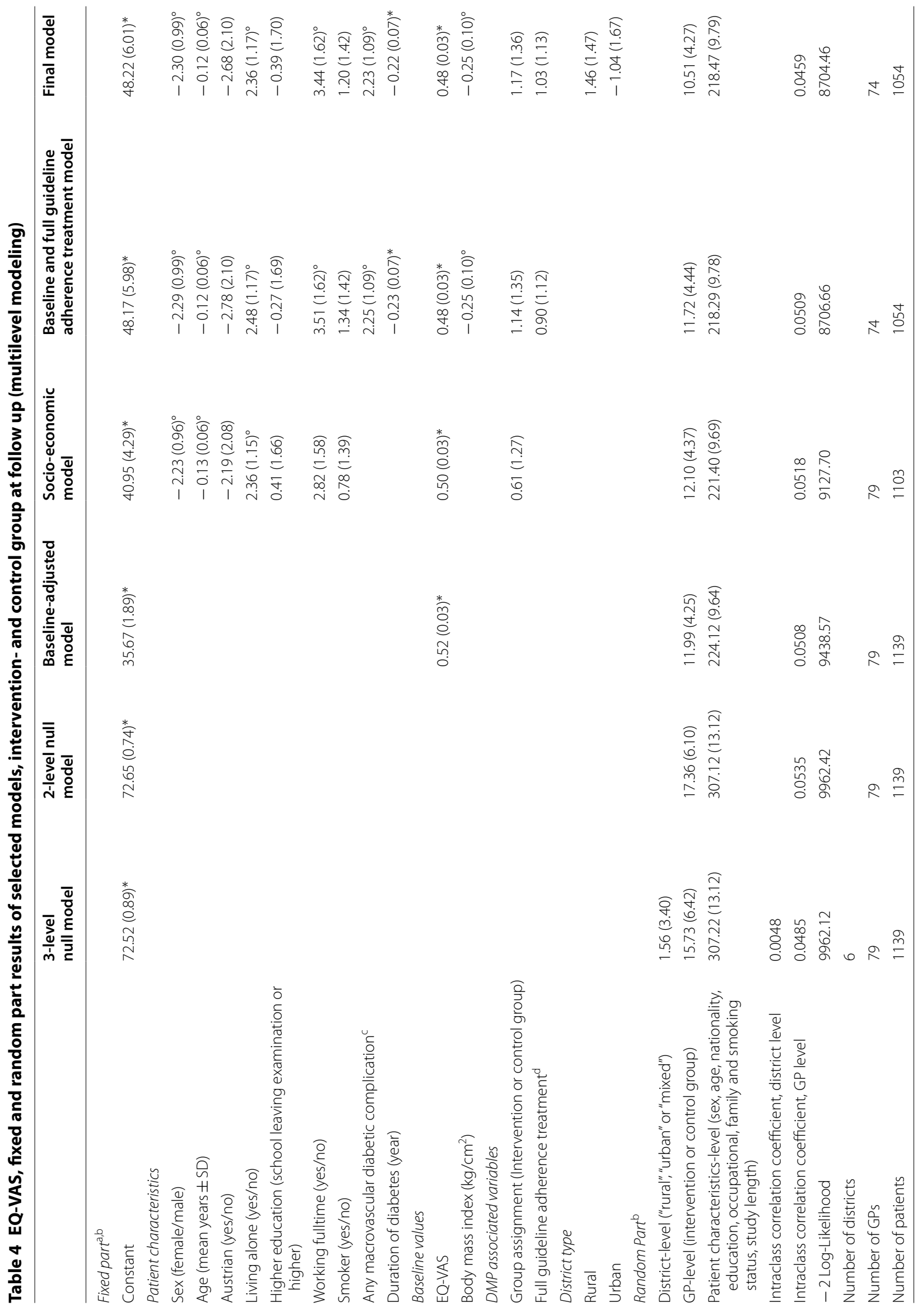




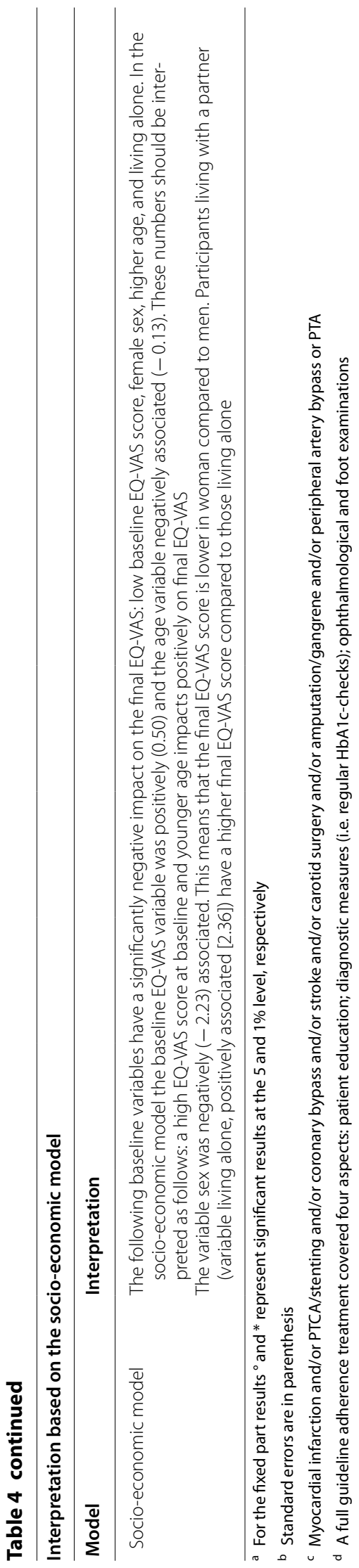




\section{Strengths and limitations}

Our trial is characterised by a high level of internal validity. Minor limitations regarding its external validity exist. As pointed out previously [15], due to a volunteer-based enrolment strategy, GPs might have recruited "healthier" and more compliant patients. Hence, it is questionable whether our sample accurately represents the population from which it was recruited. However, this might also reflect healthier patient characteristics in primary care settings.

We are aware of the limitations of the EuroQol tool (EQ-5D-3L) and of its use to evaluate HRQoL. Some researchers point out that the EQ-5D-3L rather measures quality of health $[6,31]$; while others have shown that it is an appropriate tool to evaluate HRQoL among others in patients with type 2 diabetes [32-34]. Furthermore, the restricted number of levels of the EQ-5D can be criticised. However in 2007, when the Austrian DMP "Therapie-Aktiv" was rolled out, the latest version of the EQ-5D (five levels) [26], was not yet available.

There is a lack of data regarding minimal important difference on EQ-VAS in diabetes patients. Minimal important difference estimates on EQ-5D VAS ranged from 8 to 12 point scores in cancer patients [35]. It is questionable if an improvement of 2.19 points in our diabetes population is clinically relevant. Twelve months followup might have been too short in order to demonstrate greater improvements and moreover we know that the HRQoL decreases with disease progression and complications $[6,7]$.

We did not assess psychosocial comorbidities like depression which can be a significant confounder regarding HRQoL, because it was not part of the regular DMPassessment of the Austrian "Therapie aktiv". We assume, though, that these comorbidities are evenly distributed between intervention and control group due to randomization and do not affect the main result of our study (i.e. lack of a significant difference regarding HRQoL between intervention and control).

We used a multilevel model to assess final EQ-VAS variation between patients, GPs and districts taking into account possible predictive factors. Most of the variation (approx. 95\%) could be observed on patient-level, whereas only little was attributable to GP (approx. 5\%) or district $(<0.1 \%)$ level. In our data, only a restricted number of GP-level characteristics was available. However, the variation on GP-level was small. Hence, adding additional variables on GP-level or district-level would not have changed the overall picture. The choice of variables used in subgroup analysis and multilevel modeling was based on our baseline-analysis, and on previous studies [5, 7-12] that identified age, sex, family and work circumstances, level of education, residential area, ethnicity, and any macrovascular diabetic complication, as possible predictive factors for HRQoL. We are well aware of the problems and limitations of exploratory subgroup analysis/multiple testing and the loss of power involved. However, we wanted to present some of the material as a possible basis for further research and for future development of DMPs.

\section{Comparison with existing literature}

A wide variety of educational, self-management, and structured care interventions with the aim to improve HRQoL in diabetic populations exists [16-18, 36-45]. Still, high quality randomized controlled trials are rare and little is known on the impact of DMPs on HRQoL [46]. Some studies demonstrated improvement in HRQoL $[16-18,36,40,43]$ and others did not [37-39, 44, 45]. Commonly, methodological and statistical heterogeneity exist (e.g. poor or missing randomisation, high dropout rates, pre-post comparisons, small sample size). Additionally, differences are present in composition and complexity of interventions. Duration of follow up and outcome measures are heterogeneous and hence difficult to compare. The effects seem to be largely dependent on the individual programme and its particular design [46].

\section{Implications for practice and future research}

A major drawback of disease management programmes is that they probably do not reach a considerable number of higher risk patients [47]. Our results suggest that further development of DMPs in the sense of especially focusing on patients' needs is necessary. Future research should focus on how to recruit high risk patients and how to promote patient-centred self-management and adherence to such a programme. There is a trend in Europe towards patient-centred self-management of diabetes in the primary care context. The professional role of diabetes specialist nurses, the need for multidisciplinary approaches and a focus on patient education emerge as fundamental principles in the design of relevant programmes [48], which should be strengthened in DMPs.

Since the Austrian DMP "Therapie-Aktiv" apparently has no significant impact on HRQoL after one year of intervention, we propose twofold: on the one hand, the intervention has to be intensified in terms of increasing patient empowerment and self-management. A promising and feasible approach to increase patient empowerment and adherence of patients enrolled in the DMP is initiating a peer support programme with regular physical activity, nutrition, psychological counselling, and diabetes relevant group discussions [49, 50]. Secondly, future programmes should try to better incorporate the needs of certain subpopulations. Our data show clearly that patients who are living alone, are of non-Austrian 
nationality, and are suffering from any macrovascular diabetic complication start off with lower EQ-VAS scores (up to five points below the average). This may also apply to other subgroups like patients with specific comorbidities (e.g. depression) not addressed in our study. Hence attention of future DMPs should especially focus on these subgroups.

\section{Conclusion}

In this cluster randomised controlled trial, the Austrian DMP "Therapie-Aktiv" showed no significant impact on EQ-VAS or EQ-index when compared to usual care although the EQ-VAS increased significantly within the intervention group. Our multilevel model to assess final EQ-VAS variation by patient-level, GPs' group assignment and district-level demonstrated that age, sex, family and occupational status, duration of diabetes, baseline BMI and baseline EQ-VAS have a significant impact on EQ-VAS at follow-up. Almost all variation was attributable to patient-level. Future research and development of DMPs should focus on subgroups with lower HRQoL, patient needs, and predictors of HRQoL in order to maintain or improve HRQoL.

\section{Key message}

- The EQ-VAS increased significantly within the intervention group and the improvement was stronger in women compared to men. This effect was not present in group comparison.

- The multilevel model demonstrated that sex, age, family and work circumstances, existence of any macrovascular diabetic complication, duration of diabetes, baseline body mass index and baseline EQVAS significantly influence EQ-VAS at follow-up.

- Future evaluations of the impact of DMPs on HRQoL in type 2 diabetes should incorporate predictors of HRQoL such as sex, age, socioeconomic status, coronary heart disease, and macrovascular diabetes complications in their analysis.

\section{Additional files}

Additional file 1. Baseline data (intention-to-treat analysis population).

Additional file 2. Baseline data disaggregated by sex (intention-to-treat analysis population).

Additional file 3. Baseline data, EQ-VAS subgroup analysis (per protocol analysis population).

Additional file 4. Baseline data, EQ-index subgroup analysis.

Additional file 5. Baseline data EQ-5 dimensions disaggregated by sex.

Additional file 6. EQ-index in the intervention group at follow-up, various subgroups disaggregated by sex.

\section{Abbreviations}

BMI: body mass index; DMP: disease management programme; GP: general practitioner; HRQoL: health related quality of life; RCT: randomized controlled trial; SGKK: Salzburger Gebietskrankenkasse (in English, the statutory public health insurance of the province of Salzburg).

\section{Authors' contributions}

SP: contributed substantially in writing this paper, performed the statistical analyses and interpretation of the data, and supervised the maintenance and quality assurance of the data. TJ: contributed substantially in writing this paper as well as in the interpretation of the data, and contributed to data acquisition. MF: contributed to writing this paper, interpretation of the data, and critically revised the manuscript. HW: contributed to the conception and design of the study, as well as data acquisition, and critically revised the manuscript. RW: contributed to the conception and design of the study, and critically revised the manuscript. ACS: developed the study idea, contributed substantially in writing the study protocol, supervised the writing of this paper, and was in charge of carrying out the study. All authors read and approved the final manuscript.

\section{Author details}

${ }^{1}$ Department of Mathematics, University of Salzburg, Hellbrunner Str. 34 5020 Salzburg, Austria. ${ }^{2}$ Institute of General Practice, Family Medicine and Preventive Medicine, Paracelsus Medical University, Salzburg, Strubergasse 21, 5020 Salzburg, Austria. ${ }^{3}$ Paris Lodron University, Kapitelgasse 4-6, 5020 Salzburg, Austria. ${ }^{4}$ Department of Internal Medicine, Wehrle-Diakonissen Hospital, Haydnstrasse 18, 5020 Salzburg, Austria. ${ }^{5}$ Paracelsus Medical University, Strubergasse 21, 5020 Salzburg, Austria. ${ }^{6}$ Institute of General Practice and Family Medicine, Witten/Herdecke University, Alfred-Herrhausen-Straße 50, 58448 Witten, Germany. ${ }^{7}$ Division of Population Health, Health Services Research and Primary Care, School of Health Sciences, University of Manchester, Oxford Rd, Manchester M13 9PL, UK.

\section{Acknowledgements}

We would like to express our gratitude to all the participating physicians of the province of Salzburg and their teams.

\section{Competing interests}

The authors declare that they have no competing interests.

\section{Availability of data and materials}

The datasets analysed in the current study are available from the corresponding author upon reasonable request. All data generated or analysed during this study are included in this published article [and its additional information files].

\section{Consent for publication}

Not applicable.

\section{Ethics approval and consent to participate}

Approval received from the Ethics Commission of the Province of Salzburg, Austria on the 28th February 2007 (ref: 415-E779/2-2007). All patients willing to participate were included in the study after informed consent according to the declaration of Helsinki was given.

\section{Funding}

This study was financed primarily by the Institute of General Practice, Family Medicine and Preventive Medicine of the Paracelsus Medical University, Salzburg. We received additional funding by the statutory public health insurance of the province of Salzburg (Salzburger Gebietskrankenkasse, SGKK), the Salzburg Savings Bank (Salzburger Sparkasse) and Roche Diagnostics Austria. We would like to thank our sponsors for these grants. The sponsors were not involved in the realisation of the study, data processing or evaluation.

\section{Publisher's Note}

Springer Nature remains neutral with regard to jurisdictional claims in published maps and institutional affiliations. 
Received: 11 January 2018 Accepted: 28 March 2018 Published online: 10 April 2018

\section{References}

1. Engelgau MM, Geiss LS, Saaddine JB, Boyle JP, Benjamin SM, Gregg EW, et al. The evolving diabetes burden in the United States. Ann Intern Med. 2004:140(11):945-50.

2. King H, Aubert RE, Herman WH. Global burden of diabetes, 1995-2025: prevalence, numerical estimates, and projections. Diabetes Care. 1998;21(9):1414-31.

3. Ofman JJ, Badamgarav E, Henning JM, Knight K, Gano AD Jr, Levan RK, et al. Does disease management improve clinical and economic outcomes in patients with chronic diseases? A systematic review. Am J Med. 2004;117(3):182-92

4. Pimouguet C, Le GM, Thiebaut R, Dartigues JF, Helmer C. Effectiveness of disease-management programs for improving diabetes care: a metaanalysis. CMAJ. 2011;183(2):E115-27

5. Rubin RR, Peyrot M. Quality of life and diabetes. Diabetes Metab Res Rev. 1999;15(3):205-18.

6. Solli O, Stavem K, Kristiansen IS. Health-related quality of life in diabetes: the associations of complications with EQ-5D scores. Health Qual Life Outcomes. 2010:8:18.

7. Wexler DJ, Grant RW, Wittenberg E, Bosch JL, Cagliero E, Delahanty L, et al. Correlates of health-related quality of life in type 2 diabetes. Diabetologia. 2006:49(7):1489-97.

8. Fortin M, Bravo G, Hudon C, Lapointe L, Almirall J, Dubois MF, et al. Relationship between multimorbidity and health-related quality of life of patients in primary care. Qual Life Res. 2006;15(1):83-91.

9. Grandy S, Fox KM. EQ-5D visual analog scale and utility index values in individuals with diabetes and at risk for diabetes: findings from the study to help improve early evaluation and management of risk factors leading to diabetes (SHIELD). Health Qual Life Outcomes. 2008;6:18.

10. Hodek JM, Ruhe A, Greiner W. Multimorbidity and health-related quality of life among elderly persons. Bundesgesundheitsblatt Gesundheitsforschung Gesundheitsschutz. 2009:52(12):1188-201.

11. Holmes J, McGill S, Kind P, Bottomley J, Gillam S, Murphy M. Healthrelated quality of life in type 2 diabetes (TARDIS-2). Value Health. 2000;3(Suppl 1):47-51.

12. Koopmanschap M. Coping with type II diabetes: the patient's perspective. Diabetologia. 2002:45(7):S18-22.

13. Peters SA, Huxley RR, Woodward M. Diabetes as a risk factor for stroke in women compared with men: a systematic review and meta-analysis of 64 cohorts, including 775,385 individuals and 12,539 strokes. Lancet. 2014:383(9933):1973-80.

14. Peters SA, Huxley RR, Woodward M. Diabetes as risk factor for incident coronary heart disease in women compared with men: a systematic review and meta-analysis of 64 cohorts including 858,507 individuals and 28,203 coronary events. Diabetologia. 2014;57(8):1542-51.

15. Sonnichsen AC, Winkler H, Flamm M, Panisch S, Kowatsch P, Klima G, et al. The effectiveness of the Austrian disease management programme for type 2 diabetes: a cluster-randomised controlled trial. BMC Fam Pract. 2010;11:86

16. Ose D, Miksch A, Urban E, Natanzon I, Szecsenyi J, Kunz CU, et al. Health related quality of life and comorbidity. A descriptive analysis comparing EQ-5D dimensions of patients in the German disease management program for type 2 diabetes and patients in routine care. BMC Health Serv Res. 2011:11:179.

17. Miksch A, Hermann K, Trieschmann J, Roelz A, Heiderhoff M, Laux G, et al. Gender-specific differences in quality of life of patients with type 2 diabetes with or without participating in DMP. Gesundheitswesen. 2008;70(4):250-5

18. Ose D, Wensing M, Szecsenyi J, Joos S, Hermann K, Miksch A. Impact of primary care-based disease management on the health-related quality of life in patients with type 2 diabetes and comorbidity. Diabetes Care. 2009;32(9):1594-6.

19. Laxy M, Stark R, Meisinger C, Kirchberger I, Heier M, von Scheidt W, et al. The effectiveness of German disease management programs (DMPs) in patients with type 2 diabetes mellitus and coronary heart disease: results from an observational longitudinal study. Diabetol Metab Syndr. 2015;7:77.

20. Mehring M, Donnachie E, Bonke FC, Werner C, Schneider A. Disease management programs for patients with type 2 diabetes mellitus in Germany: a longitudinal population-based descriptive study. Diabetol Metab Syndr. 2017;9:37.

21. Sonnichsen AC, Rinnerberger A, Url MG, Winkler H, Kowatsch P, Klima G, et al. Effectiveness of the Austrian disease-management-programme for type 2 diabetes: study protocol of a cluster-randomized controlled trial. Trials. 2008;9:38

22. The disease management programme for type 2 diabetes in Austria. http://www.therapie-aktiv.at. Accessed 23 Sep 2014.

23. Weitgasser R. Diabetesschulung, "Neu" für Patienten mit Typ-2-Diabetes im Land Salzburg. Diabetes Stoffwechsel und Herz. 2009;18:25-7.

24. Austrian Diabetes Association. Diabetes mellitus-guidelines for the practice. Revised and expanded 2007 edition. Wien Klin Wochenschr. 2009;121(Suppl 5):S1-87.

25. EuroQol Group. EuroQol-a new facility for the measurement of health-related quality of life. The EuroQol Group. Health Policy. 1990;16(3):199-208.

26. Rabin R, Oemar M, Oppe M. EQ-5D-3L User Guide. Basic information on how to use the EQ-5D-3L instrument. [Version 4.0]. Rotterdam: EuroOol Group; 2011.

27. Brundage M, Bass B, Davidson J, Queenan J, Bezjak A, Ringash J, et al. Patterns of reporting health-related quality of life outcomes in randomized clinical trials: implications for clinicians and quality of life researchers. Qual Life Res. 2011:20(5):653-64.

28. MLwinN Version 2.1. [computer program]. Version 2.1. 2009.

29. Flamm M, Panisch S, Winkler H, Sonnichsen AC. Impact of a randomized control group on perceived effectiveness of a Disease Management Programme for diabetes type 2. Eur J Public Health. 2012;22(5):625-9.

30. Rakovac I, Plank J, Jeitler K, Beck P, Seereiner S, Mrak P, et al. Health status of type 2 diabetics in Austria-perspective of a quality improvement initiative. Wien Med Wochenschr. 2009;159(5-6):126-33.

31. Speight J, Reaney MD, Barnard KD. Not all roads lead to Rome-a review of quality of life measurement in adults with diabetes. Diabet Med. 2009;26(4):315-27.

32. Glasziou P, Alexander J, Beller E, Clarke P. Which health-related quality of life score? A comparison of alternative utility measures in patients with Type 2 diabetes in the ADVANCE trial. Health Qual Life Outcomes. 2007;5:21.

33. Matza LS, Boye KS, Yurgin N. Validation of two generic patient-reported outcome measures in patients with type 2 diabetes. Health Qual Life Outcomes. 2007:5:47.

34. Rabin R, de Charro F. EQ-5D: a measure of health status from the EuroQol Group. Ann Med. 2001:33(5):337-43.

35. Pickard AS, Neary MP, Cella D. Estimation of minimally important differences in EQ-5D utility and VAS scores in cancer. Health Qual Life Outcomes. 2007:5:70.

36. Chaveepojnkamjorn W, Pichainarong N, Schelp FP, Mahaweerawat U. A randomized controlled trial to improve the quality of life of type 2 diabetic patients using a self-help group program. Southeast Asian J Trop Med Public Health. 2009:40(1):169-76.

37. Olivarius NF, Beck-Nielsen $\mathrm{H}$, Andreasen AH, Horder M, Pedersen PA. Randomised controlled trial of structured personal care of type 2 diabetes mellitus. BMJ. 2001;323(7319):970-5.

38. Davies MJ, Heller S, Skinner TC, Campbell MJ, Carey ME, Cradock S, et al. Effectiveness of the diabetes education and self management for ongoing and newly diagnosed (DESMOND) programme for people with newly diagnosed type 2 diabetes: cluster randomised controlled trial. BMJ. 2008:336(7642):491-5.

39. Hill-Briggs F, Gary TL, Baptiste-Roberts K, Brancati FL. Thirty-six-item short-form outcomes following a randomized controlled trial in type 2 diabetes. Diabetes Care. 2005:28(2):443-4

40. Sturt JA, Whitlock S, Fox C, Hearnshaw H, Farmer AJ, Wakelin M, et al. Effects of the diabetes manual 1:1 structured education in primary care. Diabet Med. 2008;25(6):722-31

41. Sullivan MD, Anderson RT, Aron D, Atkinson HH, Bastien A, Chen GJ, et al. Health-related quality of life and cost-effectiveness components of the action to control cardiovascular risk in diabetes (ACCORD) trial: rationale and design. Am J Cardiol. 2007;99(12A):90i-102i. 
42. Tankova T, Dakovska G, Koev D. Education and quality of life in diabetic patients. Patient Educ Couns. 2004;53(3):285-90.

43. Trento M, Passera P, Borgo E, Tomalino M, Bajardi M, Cavallo F, et al. A 5-year randomized controlled study of learning, problem solving ability, and quality of life modifications in people with type 2 diabetes managed by group care. Diabetes Care. 2004;27(3):670-5.

44. Tuthill A, Quinn A, McColgan D, McKenna M, O'Shea D, McKenna TJ. A prospective randomized controlled trial of lifestyle intervention on quality of life and cardiovascular risk score in patients with obesity and type 2 diabetes. Diabetes Obes Metab. 2007;9(6):917-9.

45. Varroud-Vial M, Simon D, Attali J, Durand-Zaleski I, Bera L, Attali C, et al. Improving glycaemic control of patients with Type 2 diabetes in a primary care setting: a French application of the Staged Diabetes Management programme. Diabet Med. 2004;21(6):592-8.

46. de Belvis AG, Pelone F, Biasco A, Ricciardi W, Volpe M. Can primary care professionals' adherence to Evidence Based Medicine tools improve quality of care in type 2 diabetes mellitus? A systematic review. Diabetes Res Clin Pract. 2009:85(2):119-31.
47. Schafer I, Kuver C, Gedrose B, von Leitner EC, Treszl A, Wegscheider K, et al. Selection effects may account for better outcomes of the German Disease Management Program for type 2 diabetes. BMC Health Serv Res. 2010;31(10):351.

48. Kousoulis AA, Patelarou E, Shea S, Foss C, Ruud Knutsen IA, Todorova E, et al. Diabetes self-management arrangements in Europe: a realist review to facilitate a project implemented in six countries. BMC Health Serv Res. 2014;2(14):453.

49. Johansson T, Keller S, Winkler H, Ostermann T, Weitgasser R, Sonnichsen AC. Effectiveness of a peer support programme versus usual care in disease management of diabetes mellitus type 2 regarding improvement of metabolic control: a cluster-randomised controlled trial. J Diabetes Res. 2016;2016:3248547.

50. Paul G, Smith SM, Whitford D, O'Kelly F, O'Dowd T. Development of a complex intervention to test the effectiveness of peer support in type 2 diabetes. BMC Health Serv Res. 2007;31(7):136.
Ready to submit your research? Choose BMC and benefit from:

- fast, convenient online submission

- thorough peer review by experienced researchers in your field

- rapid publication on acceptance

- support for research data, including large and complex data types

- gold Open Access which fosters wider collaboration and increased citations

- maximum visibility for your research: over $100 \mathrm{M}$ website views per year

At BMC, research is always in progress.

Learn more biomedcentral.com/submissions 